\title{
An Overview of Enzyme Immunoassay: The Test Generation Assay in HIV/ AIDS Testing
}

Sushanta Kumar Barik ${ }^{1 *}$, Keshar Kunja Mohanty ${ }^{1}$, Deepa Bisht ${ }^{1}$, Beenu Joshi ${ }^{1}$, Srikanta Jena ${ }^{3}$ and Srikanth Prasad Tripathy ${ }^{2}$

${ }^{1}$ National JALMA Institute for Leprosy and Other Mycobacterial Diseases, Indian Council of Medical Research, Taj-Ganj, Agra, Uttar-Pradesh, India

${ }^{2}$ National Institute for Research in Tuberculosis, Indian Council of Medical Research, Chetpet, Chennai, Uttar-Pradesh, India

${ }^{3}$ Ravenshaw University, Cuttack, Odisha, India

\begin{abstract}
In Acquired Immunodeficiency Syndrome (AIDS) routine surveillance system, it is required to identify the persons infected with Human Immunodeficiency Virus (HIV) recently or showing the clinical stages of AIDS. The sensitive and specific of the assay is essential to detect the HIV infections in early period. Human Immunodeficiency Virus (HIV) screening assay is a type of enzyme immunoassay (EIA) has gone through improvement in several generations effectively narrow the window period. The HIV specific antibodies, viral antigens are produced up to detectable level. The time is variable in different individuals to produce the HIV antibodies in the presence of the host's immune pressure. This assay was developed from first generation to fifth generation based on its sensitivity and specificity. Due to the false positive reactivity, the accurate sensitive assay is required in field validations and routine testing of HIV infected samples. This EIA is generally used as a screening assay for blood donors and individuals those are at a risk in Acquired Immunodeficiency Syndromes (AIDS). At present, the several types of EIA are the most widely used in serological test for HIV antibodies detection.
\end{abstract}

Keywords: Human immunodeficiency virus; Acquired immunodeficiency syndrome; Enzyme immunoassay; Enzyme linked immunosorbent assay; First generation enzyme immunoassay; Second generation enzyme immunoassay; Third generation enzyme immunoassay; Fourth generation enzyme immunoassay; Fifth generation enzyme immunoassay

\section{Introduction}

Acquired Immunodeficiency Syndrome (AIDS) is threatening in human population. The main cause of AIDS is due to human immunodeficiency virus. The first six cases of AIDS were reported in USA in the year 1981 [1]. HIV-1 and HIV-2 strains are exhibiting genetic diversity within and among the hosts [2]. HIV-1 is sub divided in to four groups ( $\mathrm{M}, \mathrm{N}, \mathrm{O}$ and $\mathrm{P}$ ) and nine groups (A to I) for HIV-2. The HIV-1 group $\mathrm{M}$ divided in to nine non recombinant subtypes (A$\mathrm{K})$, circulating and unique recombinant forms [3].

Human immunodeficiency virus mainly disorders the immune system of the human being [4]. The behavior changes was observed after notification of human immunodeficiency virus infection. The human immunodeficiency virus testing is essential for a person in primary infection led to engage in "high risk" activity [5]. HIV is mainly circulating in different groups of people like injecting drug users, migrants, truckers, female sex workers, male sex workers etc. and was detected by the several HIV test kits [6]. The diagnosis of HIV infection is based on the basis of serological test for detection of antibodies. The antibodies were developed during the infectious seroconversion period called "window period" and the generation of enzyme immunoassays was developed by several companies based on the window period. The time of detection of HIV antibodies of first and second generation assay was 45-60 days as well as third and fourth generation assay was 20-25 days after infection [7].The latest fifth generation assay was developed to shorten the window period up to one week of post infection.

The EIA was first invented by the research group of Peter Perlman and Eva Engvall at Stockholm University in Sweden and another research group of Anton Schuurs and Bauke van Weeman in the Netherlands [8]. In the year 1988, the World Health Organization (WHO) global program on AIDS initiated a program on commercially available assays for detecting both types of HIV antibodies like HIV-1 and HIV-2. In the year 1985, the FDA was approved the first enzyme linked immunosorbent assay for screening of blood of HIV infected patients. The HIV test like ELISA, rapid test assay, immunofluorescent assay, western blot, line immunoassay, radio immuno precipitation assay, polymerase chain reaction are being carried out in several laboratories and clinics [9]. In the early course of HIV infection, the western blot or indirect immunofluorescence assay produced false or indeterminate results. Nucleic acid amplification test (NAT) has been used to check the primary infection. This method was a very sensitive method for detection of HIV antibodies in high risk population [10]. Among the test assays, EIA or ELISA is the best sensitive and specific assay used by several laboratories and clinics in the world to detect HIV infection.

The first generation to fifth generation assay was developed by different companies based on the sensitivity and specificity of the assay to detect the HIV variants. A variety of EIA automated and manual methods are manufactured by several companies in the world. This is generally used to detect the serum, urine, oral fluid samples of HIV infected patients in a good resource laboratory facility.

\section{Types of the HIV test generation assays}

EIA has been currently used in all laboratories to detect HIV infection within the window period. EIA is mainly used for the initial

*Corresponding author: Sushanta Kumar Barik, National JALMA Institute for Leprosy and Other Mycobacterial Diseases, Taj-Ganj, Agra-282001, India, Tel: +919411651219; E-mail: sushantakumarbarik82@gmail.com

Received February 12, 2018; Accepted March 19, 2018; Published March 26 , 2018

Citation: Barik SK, Mohanty KK, Bisht D, Joshi B, Jena S, et al. (2018) An Overview of Enzyme Immunoassay: The Test Generation Assay in HIVIAIDS Testing. J AIDS Clin Res 9: 762. doi: 10.4172/2155-6113.1000762

Copyright: ( 2018 Barik SK, et al. This is an open-access article distributed unde the terms of the Creative Commons Attribution License, which permits unrestricted use, distribution, and reproduction in any medium, provided the original author and source are credited. 
evaluation of established HIV infection. EIA is based on the principle of antigen-antibody reaction. In the first generation assay, the whole virus was used as antigens, prepared from cell culture. In the first generation enzyme immunoassay, the anti-HIV IgG antibodies were detected. The first generation assay is unable to detect the HIV-1 antigens. The second generation assay used either recombinant /synthetic peptide antigens instead of viral lysate that would react with anti-HIV antibodies. The second generation assay detected only the IgG antibody. The third generation assay used for detection of both HIV-1/HIV-2 IgG and IgM antibodies. The fourth generation assay detects p24 antigen, HIV$1 /$ HIV-2 antibodies in a single reaction plate [11].The recent FDA approved fifth generation assay differentiates the HIV-1 antibodies, HIV-2 antibodies and HIV-1 p24 antigen in serum and plasma samples.

\section{First generation enzyme immuno assay}

The first generation EIA was developed for detection of AIDS in the year 1985. The antibodies were detected to bind to HIV antigens produced by cell culture in indirect approach. The first generation EIA was enough sensitive but less effective in specificity. The first generation EIA remains very widely used in both clinical and health laboratories due to their low cost and reliability. This first generation EIA detected only the M-group specific HIV antigens not detect the Non M-subgroup and HIV-2 antigens. As this first generation assay used the cell lysate antigens had frequently nonspecific reactions between antibodies and antigens, the western blot analysis is a confirmatory method for the first generation tested samples. The first generation EIA was used for blood bank screening program [12].

This first generation EIA was developed by very less number of companies like Abbott, Vironostika. The Abbott HTLV-III first generation ELISA specificity was tested and then used to detect the HIV infection in several laboratories in the world [13]. An IgG sensitive HIV antibody test assay was developed by Vironostika HIV-1 microelisa system (viral lysate) ${ }^{\circ}$ (biomerieux, Durham, NC) used to detect the acute HIV infections in high risk patients in California [14].

\section{Second generation enzyme immuno assay}

The second generation EIA was developed in the year 1987. This assay is the indirect format like the first generation assay. In the second generation EIA, the HIV recombinant antigens and peptides was used instead of whole viral lysate bound in solid phase. The recombinant antigens increased the specificity and sensitivity of the test being reduced the window period to 33-35 days of post infection. The second generation EIA was used in blood banks because this test reduces the risk of post transfusion HIV infection [15].

The second generation assay was developed by recombinant DNA technology or synthetic peptide antigens $\mathrm{M}, \mathrm{N}, \mathrm{O}$ group. A panel of known positive sera and blood donors were tested with two competitive screening assays. (Behring and Wellcozyme company) and two second generation assays using antigens generated by recombinant DNA technology (Abbott) and synthetic peptides assay (Biochrom).The sensitivity and specificity of the second generation Abbott assay was the best choice for blood screening purposes [16].

The highly sensitive second generation EIA made by three manufacturers (Abbott laboratories, Abbott park III, Organon Technika Corp., Belgium and Well Come Foundation, UK') was better than the confirmatory tests like western blot (DuPont Biotech, USA), Radio immuno precipitation assay, (RIPA,CLB), Competitive immunoassay (Abbott laboratories, Abbott park III). The early detection of HIV antibodies was tested by the second generation assay (Vidas HIV $-1+2$ assay") and the specificity was tested from sera in a low risk population [17].

The second generation assay was no more used in the laboratories because of more cross reactivity of antibodies with the group $M$ subtypes.

\section{Third generation enzyme immune assay}

The Third generation EIA was developed in the year 1994.The third generation EIA was designed in a sandwich format. Recombinant HIV1 and HIV-2 proteins/peptides bound on the solid phase of the micro plate to react with patient serum. This third generation EIA test reduced drastically the window period to about 22 days of post infection. This assay is very sensitive as it detects both IgG and IgM antibodies in HIV infected serum. The third generation assay was used to detect in early stages of HIV infection [18].

Many research papers have been published on the sensitivity and specificity of the third generation assay by comparing kits of different companies in a uniform serum samples. The highest sensitive test is always considered to be good in comparing with other test kit companies. The LG Anti-HIV 1/2 Plus ELISA (LG life Sciences, Seoul, Korea), a new third generation enzyme linked immune sorbent assay was compared to the Enzygnost Anti-HIV 1/2 Plus ELISA (Dade Behring, Marburg, Germany) was shown to be $100 \%$ sensitivity but the specificity of the LG Anti-HIV 1/2 Plus ELISA was more than the Enzygnost Anti-HIV 1/2 Plus ELISA ${ }^{\circ}$ as it was detected the highly divergent subtypes including HIV-1 group O [19].

The third generation assay was developed to decrease the number of false negative results. A panel of sero-conversion HIV antibodies was tested by Vidas biomérieux HIV $1 / 2^{\circ}$ (second generation ELISA), Murex HIV $1-2^{\circ}$ (third generation ELISA) as well as with western blotting and radio immuno western blotting. The performance of the Murex HIV $1 / 2^{\circ}$ third generation assay was the best among all the assays [20].

The HIV group specific third generation assay was developed, tested and so called "Plus O assay". The current version of an anti-HIV-1/ anti-HIV-2 third generation assay was developed by Vironostika HIV Uni-Form II, an HIV-O specific peptide was derived from immuno dominant region of HIV-1 group O gp41 strain ANT 70 was introduced in order to improve the HIV-1 group reactivity. This assay was used to check the group $\mathrm{O}$ virus found in two persons of Cameroonian origin [21].

The superiority and specificity of different generations assay was determined by a comparative algorithm. The sensitivity and typespecificity was compared by an algorithm combining a third generation EIA followed by a confirmatory multi-spot assay with the conventional algorithm combines with a third generation EIA Bio-Rad GS HIV-1/ HIV-2 Plus $\mathrm{O}^{\circ}$ enzyme immuno assay with a confirmatory western blot Bio-Rad GS HIV-1 WB' [22]. In case of the detection of HIV antibodies in oral fluids by third generation EIA has been possess superior sensitivity than the first and second generation assays [23].

The third generation EIA was used to detect the vaccine induced antibodies and true infections in acquired HIV patients. HIV Selectest an EIA containing p6 and gp41 peptides of HIV. This Selectest assay was used mainly to detect the vaccine induced antibodies in men and women serum [24].

This third generation EIA is now used in screening purposes of the many laboratories of developing countries. 


\section{Fourth generation enzyme immuno assay}

The fourth generation EIA was developed in the year 1997. Fourth generation EIA was developed to reduce the diagnostic window period. The fourth generation assay detects the HIV p24 antigen with anti HIV IgG and IgM antibodies. HIV p24 antigen was detected about 2-18 days in HIV infected patients before seroconversion. Sometimes, the early detection of HIVp24 core protein is depends on the humoral immuno response of the host. Sometimes, the second diagnostic window period is non-reactive due to absence of the HIV specific antibodies and depletion of p24 antigen concentration [25].

The sensitivity and specificity of the fourth generation assays developed by different companies is directly correlated with copy number of the p24 HIV antigen in the window period. Some of the fourth generation assay was reactive in the first diagnostic window period where as nonreactive in second diagnostic window period and vice-versa. The fourth generation AxSYM HIV Ag/Ab Combo assay (Abbott Laboratories, Abbott Park, Ill.) was very strong reactive in a four days HIV infected patient but the AxSYM HIV-1/2 Antibody gO assay (Abbott Laboratory, Abbott Park, Ill.) was nonreactive in the same. The HIV p24 antigen EIA, Vironostika HIV-1 antigen EIA', bioMeriaux;, Boxtel, Netherlands was reactive on nine days serum samples but was non-reactive in AxSYM HIV Ag/Ab Combo assay and AxSYM HIV$1 / 2$ Antibody gO assay. The manufacturers of fourth generation assay sensitivity was based on the copy number of the p 24 antigen in first and second diagnostic window period as well as the condition of the host immune system [26].

The fourth generation VIDAS HIV DUO Ultra (Biomérieux, Marcy-l'Etoile, France) enzyme linked fluorescent ELISA was detected the p24 antigen and HIV-1 (with group O) and HIV-2 antibodies. The VIDAS HIV DUO Ultra was shown a comparable sensitivity among the assay manufacturers like Enzygnost HIV Integral', Genscreen HIV $1 / 2$ version $2^{\circ}$ (third-generation EIA), Genscreen Plus HIV Ag-Ab and Genetic Systems HIV-1 Ag EIA (p24 antigen assay), Enzymun-Test HIV Combi" with 15 cell culture supernatants infected with different HIV-1 subtypes and 257 potentially cross-reactive serum samples, 16 sero-conversion panels. The VIDAS HIV DUO Ultra reduced the diagnostic window period by 3.82 days. The VIDAS HIV DUO Ultra was proven the best test assay for blood donors [27].

The main aim of the fourth generation assay test is to detect acute HIV infections, to know about the percentage of HIV status in low and high risk population. The Bio-Rad GS HIV Combo Ag/Ab EIA was based on the detection of the HIVp24 antigen and antibodies to HIV-1 (group O and group M) and HIV-2 in human serum or plasma. The GS combo Ag/Ab assay reduced the diagnostic window period as compared with the third generation assay [28].

The fourth generation HIV test has the potential advantages over conventional HIV testing for time and cost saving identifying both early and established HIV infections. The above VIDAS HIVDUO Ultra (BioMe'rieux, Marcy l'Etoile, France) is claimed to be more sensitive in screening test for both early and established infections. The performance of DUO Ultra was challenged with sera of 2838 samples from populations. The HIV DUO Ultra was shown $100 \%$ sensitivity and $99.5 \%$ specificity above tested samples [29].

The combination of both antigen and antibody detection increases sensitivity in the early sero-conversion, improving the chances of identification of low-titer anti-HIV antibodies in the late stage of infection. The two principles are combined in one assay. So, the fourthgeneration assays have been reported to possess potential non-specific reactivity. Therefore, the early diagnosis of HIV reduces mortality and morbidity. A comparison was made in between third and fourth generation assay of the same company and the fourth generation assay found to be more sensitive. A study was conducted based on the performance of a new fourth-generation ADVIA Centaur HIV antigen/ antibody combo assay with the third generation ADVIA centaur HIV $1 / \mathrm{O} / 2$ enhanced assay (EHIV) ${ }^{\circ}$ (Siemens Healthcare Diagnostics Inc, USA ) for early detection of HIV infection. This assay reduces the false positive rates [30].

The detection of current circulating HIV strains is very essential. The fourth generation Abbott Architect HIV Ag/Ab combo chemiluminescent magnetic microparticle based immunoassay was used to detect early HIV infections of serum specimens in San Diego County along with the DiaSorin Liaison XL Murex HIV Ab/Ag assay but the clinical sensitivity (100\%) and specificity (98.9\%) of the new Dia Sorin Liaison XL Murex HIV Ab/Ag assay was more compared with the Abbott Architect HIV Ag/Ab Combo kit when tested with 900 samples including negative samples (493) and positive samples(407) representatives of HIV-1 group M subtypes, Group O subtypes, HIV-2 variants and Circulating Recombinant Forms (CRFs) in France [31].

In a specialized clinical setting, a better sensitive and specific fourth generation assay is required for case finding, description of virological, epidemiological and clinical characteristics of acute HIV patients. Diagnosis of acute stages of HIV infection is necessary to challenge with the virus replication followed by immediate combined antiretroviral therapy initiation. During the acute stage of HIV infection, the retrospective study with confirmatory testing is essential. To consider the $100 \%$ confirmatory results of the acute HIV infected samples, then have to make the test with several assays. The serum samples reactive with a fourth generation HIV-1/2 assay (Architect HIV Ag/Ab Combo, Abbott) was then retested with another fourth generation assay (VIDAS DUO HIV Ultra", Biomeriux), Western blot assay" (New Lav-1 Biorad), Geenius confirmatory assay" (Biorad) [32].

In case of the serological status monitoring of AIDS patients, the fourth generation ELISA Vironostika HIV UniForm II Ag/Ab assay was well established and shown to be good sensitive for both HIV-1 and HIV-2 [33]. The performance of the GS Biorad fourth generation HIV$1 / 2$ immunoassay demonstrated high sensitivity and specificity for both early and established infections as well as high specificity in a low HIV population. This Birorad GS fourth generation assay was a superior assay than the third generation, multispot, western blot as well as nucleic acid test. Now, this Biorad GS fourth generation assay is widely used in most of the laboratory [34]. The fourth generation assay is the most advance assay for detection of viral antigens and antibodies of several genetic diversity groups of HIV [22]. Fourth generation Ag/Ab immunoassay like Roche Elecsys @HIV combi PT and Diasorin Liaison XL Murex Ag/Ab assay was performed on dried serum spot collected by filter paper had good performance for early infection of HIV patients [35].

HIV point care of testing is essential to generate result quickly in an economical and clinical effectiveness. The fourth generation Alere Determine HIV-1/2 Ag/Ab Combo POCT was shown $88 \%$ sensitivity and $100 \%$ specificity reacted with acute HIV infected samples [36].

In case of the large scale screening of blood donors and clinical samples, the automated fourth generation enzyme immuno assay is essential.

\section{Fifth generation enzyme immuno assay}

In the year 2015, the fifth generation assay was developed. The Food 
and Drug Administration was approved the Bio-Rad Bioplex $2200 \mathrm{HIV}$ $\mathrm{Ag} / \mathrm{Ab}$ for screening the HIV. The fifth generation assay detects both HIV-1 antibody and HIV-1 p24 antigen but provides separate results for HIV-1 and HIV-2 antibody [37]. The fifth generation assay is more advantages than the fourth generation assay due to multiplexed screening test. This fifth generation assay detects and differentiates all three HIV markers like HIV-1 antibodies, HIV-2 antibodies and HIV-1 p24 antigen.

\section{General Features in HIV Testing}

The key features of HIV testing are discussed below:

- The HIV testing is the main risk and responsibility of life of a person in social busy schedule.

- The clinical course would not be changed due to delayed in HIV testing in a high risk population groups like injecting drug users, sex workers, truckers, etc.

- HIV testing further reduces the number of potential infections and early health care.

- HIV testing prevent mother to child transmission in a population level and prevent the early management of opportunistic infections.

- HIV testing is essential for women during pregnancy.

- Even the probability of HIV infected population is low in a community but the HIV testing should be essential.

- The highly sensitive and specific HIV testing assay reduced the mortality and morbidity in a population level.

- To minimize the cost effectiveness and time, a suitable test generation assay is recommended.

- HIV testing with the latest generation EIA is essential with the informed consent.

- People living with affordable and high quality life, HIV testing is essential to ensure the working condition.

- The excellent test generation assay reduces the consequences activities among the high risk group of people.

- The promotion of the latest test generation assay in each house hold is the main goal of HIV prevention.

- The HIV testing should recommend in the HIV infected person's bearing the signs, symptoms or illness.

- Early detection with latest generation assay is very essential for each individual that can be leads to acute infection.

- The mode of HIV transmission risk is higher in the patients with early or acute infection rather than established infection. So, the suitable testing generation is essential.

- Latest generation testing assay is essential in seroprevalence study in a HIV infected population.

- The detection of HIV infection can reduce the risky behavior of HIV infected patients. The effective care can reduce the morbidity, mortality and viral load.

\section{Conclusion}

The early detection of HIV generated antibodies in the body of human being needs to develop the latest generation assay. The mandatory of the advance generation assay is required in a good laboratory practice, laboratory research or the diagnostic research. When a generation assay will develop by a researcher or a company, the performance of the assay may be higher or lower depends upon the reactivity and specificity of the developed assay. The recent generation EIA carrying properties like sensitivity and specificity developed by the reputed companies those are involved with diagnostic research in the area of human immunodeficiency virus.

\section{Acknowledgement}

Thanks to Indian Council of Medical Research, Govt. of India for providing the senior research fellowship in the area of HIV research.

\section{References}

1. Greene WC (2007) A history of AIDS: looking back to see ahead. Eur J Immuno 37: S94-102.

2. Sharp PM, Hahn BH (2010) The evolution of HIV-1 and the origin of AIDS Philos Trans R Soc Lond B Biol Sci 365: 2487-2494.

3. Taylor BS, Sobieszczyk ME, McCutchan FE, Hammer SM (2008) The challenge of HIV-1 subtype diversity. N Engl J Med 358: 1590-1602.

4. Lee SH, Sheon N (2008) Responsibility and risk: Accounts of reasons for seeking an HIV test. Sociol Health IIIn 30: 167-181.

5. Cleary PD, Van Devanter N, Rogers TF, Singer E, Shipton-Levy R, et al. (1991) Behavior changes after notification of HIV infection. Am J Public Health 81 1586-1590.

6. Delaney KP, Heffelfinger JD, Wesolowski LG, Owen SM, Meyer WA, et al. (2011) Performance of an alternative laboratory-based algorithm for HIV diagnosis in a high-risk population. J Clin Virol 52: S5-S10.

7. Branson BM (2010) The future of HIV testing. J Acquir Immune Defic Syndr 55: S102-S105.

8. Lequin RM (2005) Enzyme immunoassay (EIA)/enzyme-linked immunosorbent assay (ELISA). Clin Chem 51: 2415-2418.

9. Cornett JK, Kirn TJ (2013) Laboratory diagnosis of HIV in adults: A review of current methods. Clin Infect Dis 57: 712-718.

10. Krajden M, Cook D, Mak A, Chu K, Chahil N, et al. (2014) Pooled nucleic acid testing increases the diagnostic yield of acute HIV infections in a high-risk population compared to $3^{\text {rd }}$ and $4^{\text {th }}$ generation HIV enzyme immunoassays. $J$ Clin Virol 61: 132-137.

11. Buttò S, Suligoi B, Fanales-Belasio E, Raimondo M (2010) Laboratory diagnostics for HIV infection. Annalidell'Istitutosuperiore di sanita 46: 24-33.

12. Branson BM (2007) State of the art for diagnosis of HIV infection. Clin Infect Dis 45: S221-S225.

13. Lequin RM (2005) Enzyme immunoassay (EIA)/enzyme-linked immunosorbent assay (ELISA). Clin Chem 51: 2415-2418.

14. Patel P, Klausner JD, Bacon OM, Liska S, Taylor M, et al. (2006) Detection of acute HIV infections in high-risk patients in California. J Acquir Immune Defic Syndr 42: 75-79.

15. Bäcker U, Gathof A, Howe J, Strobel E, Deinhardt F, et al. (1988) A new secondgeneration anti-HIV-1 enzyme immunoassay using recombinant envelope and core proteins. AIDS 2: 477-480.

16. Maskill WJ, Crofts N, Waldman E, Healey DS, Howard TS, et al. (1988) An evaluation of competitive and second generation ELISA screening tests for antibody to HIV. J Virol Methods 22: 61-73.

17. Lelie PN, Reesink HW, Huisman $\mathrm{H}$ (1988) Evaluation of three second-generation and three confirmatory assays for antibodies to human immunodeficiency virus. Voxsanguinis 54: 84-91.

18. Zaaijer HL, Exel-Oehlers PV, Kraaijeveld T, Altena E, Lelie PN (1992) Early detection of antibodies to HIV-1 by third-generation assays. Lancet 340: 770 772

19. Yeom JS, Lee JB, Ryu SH, Kang HJ, Kim S, et al. (2006) Evaluation of a new third-generation ELISA for the detection of HIV infection. Ann Clin Lab Sci 36: 73-78 
Citation: Barik SK, Mohanty KK, Bisht D, Joshi B, Jena S, et al. (2018) An Overview of Enzyme Immunoassay: The Test Generation Assay in HIV/ AIDS Testing. J AIDS Clin Res 9: 762. doi: 10.4172/2155-6113.1000762

Page 5 of 5

20. Portincasa P, Conti G, Zannino T, Visalli S, Chezzi C (1994) Radioimmune western blotting in comparison with conventional western blotting, second and third generation ELISA assays for the serodiagnosis of HIV-1 infection. $\mathrm{N}$ Microbiol 17: 169-176.

21. Van Binsbergen J, Keur W, vdGraaf M, Siebelink A, Jacobs A, et al. (1997) Reactivity of a new HIV-1 group O third generation A-HIV-1/-2 assay with an unusual HIV-1 seroconversion panel and HIV-1 group O/group M subtyped samples. J Virol Methods 69: 29-37.

22. Torian LV, Forgione LA, Punsalang AE, Pirillo RE, Oleszko WR (2011) Comparison of multi-spot EIA with Western blot for confirmatory serodiagnosis of HIV. J Clin Virol 52: S41-S44.

23. Louie B, Lei J, Liska S, Dowling T, Pandori MW (2009) Assessment of sensitivity and specificity of first, second, and third generation EIA for the detection of antibodies to HIV-1 in oral fluid. J Virol Methods 159: 119-121.

24. Khurana S, Norris PJ, Busch MP, Haynes BF, Park S, et al. (2010) HIV-selectest enzyme immunoassay and rapid test: Ability to detect seroconversion following HIV-1 infection. J Clin Microbiol 48: 281-285

25. Vallefuoco L, Mazzarella C, Portella G (2016) Fourth generation assays for HIV testing. Exp Rev Mol Diagn 16: 723-732.

26. Speers D, Phillips P, Dyer J (2005) Combination assay detecting both human immunodeficiency virus (HIV) p24 antigen and anti-HIV antibodies opens a second diagnostic window. J Clin Microbiol 43: 5397-5399.

27. Weber B, Berger A, Rabenau H, Doerr HW (2002) Evaluation of a new combined antigen and antibody human immunodeficiency virus screening assay, VIDAS HIV DUO Ultra. J Clin Microbiol 40: 1420-1406.

28. Bentsen C, McLaughlin L, Mitchell E, Ferrera C, Liska S, et al. (2011) Performance evaluation of the Bio-Rad Laboratories GS HIV Combo Ag/Ab EIA, a 4th generation HIV assay for the simultaneous detection of HIV p24 antigen and antibodies to HIV-1 (groups M and O) and HIV-2 in human serum or plasma. J Clin Virol 52: S57-61.
29. Saville RD, Constantine NT, Cleghorn FR, Jack N, Bartholomew C, et al. (2001) Fourth-generation enzyme-linked immunosorbent assay for the simultaneous detection of human immunodeficiency virus antigen and antibody. J Clin Microbiol 39: 2518-2524.

30. Lee K, Park HD, Kang ES. Reduction of the HIV seroconversion window period and false positive rate by using ADVIA Centaur HIV antigen/antibody combo assay. Ann Lab Med 33: 420-425.

31. Lemee V, Leoz M, Etienne M, De Oliveira F, Plantier JC (2014) Performance of the Liaison XL Murex HIV Ab/Ag test on clinical samples representing current epidemic HIV variants. J Clin Microbiol 52: 3277-3279.

32. Ammassari A, Abbate I, Orchi N, Pinnetti C, Rozera G, Et al. (2014) Acute HIV infection (AHI) in a specialized clinical setting: Case-finding, description of virological, epidemiological and clinical characteristics. J Int AIDS Soc 17 19676.

33. Sudha T, Teja VD, Gopal M, Rajesh M, Lakshmi V (2005) Comparative evaluation of TRI-DOT Rapid HIV test with fourth-generation ELISA for the detection of human immunodeficiency virus. Clin Microbiol Infect 11: 850-852.

34. Nasrullah M, Wesolowski LG, Meyer III WA, Owen SM, Masciotra S, et al. (2013) Performance of a fourth-generation HIV screening assay and an alternative HIV diagnostic testing algorithm. AIDS 27: 731-737.

35. Kania D, Truong TN, Montoya A, Nagot N, Van de Perre P, et al. (2015) Performances of fourth generation HIV antigen/antibody assays on filter paper for detection of early HIV infections. J Clin Virol 62: 92-97.

36. Fitzgerald N, Cross M, O'Shea S, Fox J (2016) Diagnosing acute HIV infection at point of care: A retrospective analysis of the sensitivity and specificity of a fourth-generation point-of-care test for detection of HIV core protein p24. Sex Transm Infect 93: 100-101.

37. https://www.medscape.com/viewarticle/848576 